\title{
Influencia del juego interior de la selección española de baloncesto en el rendimiento: análisis de coordenadas polares.
}

\section{The influence of Spanish basketball team inside game in performance: polar coordinates analysis.}

\section{Influência do jogo interno da selecção nacional de basquetebol espanhola no desempenho: análise das coordenadas polares.}

\author{
Morillo-Baro, J.P ${ }^{1}$, Troyano-Gallegos, B ${ }^{1}$, Alejandre Estable, A ${ }^{1}$, Vázquez-Diz, J.A ${ }^{1}$, Reigal Garrido, \\ R.E ${ }^{1}$, Hernández-Mendo, $\mathrm{A}^{1}$ y Morales-Sánchez, $\mathrm{V}^{1}$. \\ ${ }^{1}$ Universidad de Málaga, Málaga (España)
}

\section{RESUMEN}

En los últimos años se ha incrementado el interés por conocer las acciones más efectivas durante la competición ya que este hecho se ha mostrado esencial para mejorar el rendimiento de los jugadores en el deporte de élite; particularmente en los deportes colectivos el análisis táctico ha crecido de forma exponencial. El objetivo de este estudio fue detectar las relaciones que se establecen entre las conductas de finalización de los jugadores interiores de un equipo de baloncesto y su repercusión en el rendimiento del equipo. Para ello, y utilizando un diseño nomotético, de seguimiento y multidimensional, se realizó un análisis de coordenadas polares con las observaciones del juego de la Selección Española de Baloncesto Senior Masculina durante su participación en el Campeonato de Europa de 2015. La herramienta de observación creada ha superado el análisis de calidad del dato exigible en Metodología Observacional y está compuesta por 5 criterios y 29 categorías exhaustivas y mutuamente excluyentes. Se registraron un total de 316 acciones ofensivas, que pusieron de manifiesto la influencia de los jugadores que ocupan las posiciones interiores en el ataque estático. Los resultados muestran una alta efectividad cuando los ataques son acabados por ellos y la alta producción ofensiva de estos jugadores en acciones individuales, siendo capaces de conseguir canastas o provocar faltas a sus defensores. El análisis de coordenadas polares se ha mostrado como una técnica útil para el análisis táctico de las conductas que suceden en el deporte de competición.

Palabras clave: Metodología observacional, Coordenadas polares, Baloncesto, Análisis táctico.

\section{ABSTRACT}

In recent years, there has been an increased interest in knowing the most effective actions during competition, as this fact has proven essential to improve the performance of players in elite sport; particularly in team sports, tactical 


\section{Morillo-Baro et al.}

analysis has grown exponentially. The objective of this study was to detect the relationships that were obtained between the finishing behaviors of the inside players of a basketball team and it is impact on team performance. To do so, and using nomothetic, follow-up and multidimensional observational design, polar coordinates analysis was performed from the observations of the game of the Spanish men basketball team during European Championships in 2015. The observational tool, formed by 5 criteria and 29 exhaustive and mutually exclusive categories, was created and passed the data quality analysis required in Observational Methodology. A total of 316 offensive actions were registered and revealed the influence of the inside players in the static attack. The results showed a high effectiveness when the attacks are finished by inside players, and the high offensive production of these players in individual actions, being able to get baskets or cause fouls to their defenders. Polar coordinates analysis has been shown as a useful technique to tactical analysis of the behaviors that occur in competitive sport.

Keywords: Observational methodology, Polar coordinates, Basketball, Tactical analysis.

\section{RESUMO}

Nos últimos anos tem havido um interesse crescente em conhecer as acções mais eficazes durante a competição, uma vez que este facto se revelou essencial para melhorar o desempenho dos jogadores no desporto de elite; particularmente nos desportos colectivos, a análise táctica tem crescido exponencialmente. O objectivo deste estudo era detectar as relações estabelecidas entre os comportamentos finais dos jogadores internos de uma equipa de basquetebol e o seu impacto no desempenho da equipa. Para o fazer, e utilizando um design nomotético, de seguimento e multidimensional, foi realizada uma análise de coordenadas polares com as observações do jogo da Selecção Espanhola de Basquetebol Masculino Sénior durante a sua participação no Campeonato Europeu de 2015. O instrumento de observação criado passou a análise da qualidade dos dados exigida na Metodologia de Observação e é composto por 5 critérios e 29 categorias exaustivas e mutuamente excludentes. Foi registado um total de 316 acções ofensivas, que mostraram a influência de jogadores que ocupavam posições internas no ataque estático. Os resultados mostram uma elevada eficácia quando os ataques são finalizados por eles e a elevada produção ofensiva destes jogadores em acções individuais, podendo obter cestos ou causar faltas aos seus defensores. A análise das coordenadas polares demonstrou ser uma técnica útil para a análise táctica dos comportamentos que ocorrem no desporto de competição.

Palavras chave Metodologia observacional, Coordenadas polares, Basquetebol, Análise táctica.

\section{INTRODUCCIÓN}

En las investigaciones publicadas sobre la planificación del entrenamiento en los deportes colectivos se destaca el aumento progresivo de las demandas físicas, técnicas y tácticas en la competición en las últimas décadas (Sarmento et al., 2017). Este hecho ha provocado la necesidad de incrementar el conocimiento específico para llevar a cabo una planificación adecuada y efectuar una preparación del equipo lo más completa posible (Nunes et al., 2015). El baloncesto es un claro ejemplo de esta circunstancia, el alto nivel de intensidad en la alta competición requiere que los jugadores que compiten presenten una preparación física óptima y un bagaje técnico-táctico amplio que les permita adaptarse a los ambientes continuamente cambiantes a los que se enfrentan. El carácter abierto que presentan los deportes colectivos le confiere un alto grado de incertidumbre (Conejero, Claver, FernándezEchevarría, González-Silva y Moreno, 2016).

$\mathrm{Al}$ igual que ocurre con las investigaciones sobre otros deportes colectivos (Ortín, Olmedilla y Lozano, 2003; Morales, Lorenzo y Concepción, 2008; Castro, 2018), en el baloncesto la mayor parte de ellas se centran en la fase del ataque estático. Numerosos ejemplos dan muestra de ello: Moreno y Gómez (2017) analizaron las rachas de lanzamiento a partir de las variables asociadas al juego en el baloncesto profesional masculino; Muñoz-Arroyave, Serna, Daza e Hileno (2015) estudiaron la influencia del bloqueo directo y el uno contra uno en el éxito del lanzamiento a través de la observación y registro de los 3 partidos que disputó el FC Barcelona durante la disputa de la Copa del Rey de la temporada 2012-2013; por su parte, Romarís, Refoyo y Coterón (2013) analizaron las finalizaciones de las posesiones mediante el estudio de las 4605 situaciones de 14 partidos de la liga ACB y 


\section{El juego interior en el rendimiento del baloncesto}

12 de la liga femenina de la temporada 2009-2010 . Otros estudios que también han analizado el ataque estático en baloncesto lo han hecho centrándose más en la toma de decisión de los jugadores; como, por ejemplo, las distintas soluciones que tienen las situaciones de bloqueo (Suárez-Cadenas, Courel y Cárdenas, 2017).

Todas estas investigaciones han sido desarrolladas bajo los parámetros que marca la Metodología Observacional (MO), ampliamente referenciada como adecuada para analizar el comportamiento en el deporte (Anguera y Hernández-Mendo, 2013). Concretamente, varias herramientas han sido creadas para analizar lo que ocurre en las situaciones de juego del baloncesto; por ejemplo, Muñoz-Arroyave y Serna (2015), construyeron una herramienta para observar la relación entre algunas acciones que tiene el jugador con balón para obtener ventajas sobre su rival (uno contra uno exterior e interior y el bloqueo directo) y el éxito en los lanzamientos y, García-Santos e Ibáñez (2016) la diseñaron con la finalidad de valorar la actuación del árbitro de baloncesto.

La MO se desarrolla en contextos naturales o habituales, y consiste en un procedimiento científico que, en función de los objetivos planteados, pone de manifiesto la ocurrencia de conductas perceptibles, para proceder a su registro organizado mediante un instrumento elaborado específicamente y utilizando los parámetros adecuados. Una técnica de análisis muy utilizada en los últimos años en el ámbito de las Ciencias del Deporte es el análisis de Coordenadas Polares (Hernández-Mendo y Anguera, 1999; Castellano y Hernández-Mendo, 2003).

Existen numerosos trabajos que la han empleado para analizar distintas modalidades deportivas colectivas, como el fútbol, balonmano o el balonmano playa. En fútbol, Castañer et al. (2016) analizaron las conductas motoras que utiliza Messi para resolver situaciones de juego justo después de recibir un pase y antes de anotar un gol. En balonmano, Quiñones et al. (2020) identificaron las conductas de éxito en el juego combinativo ofensivo diferenciando entre la categoría masculina y femenina. En balonmano playa, VázquezDiz et al. (2019a) analizaron la toma de decisión del jugador especialista a través del rastro de sus conductas, y estos mismos autores (Vázquez-Diz et al., 2019b) también observaron la incidencia de los factores contextuales sobre la toma de decisión de los jugadores de balonmano playa.

Específicamente en baloncesto, destaca el estudio de Nunes et al. (2015) donde se estudian las acciones de pick and roll y su influencia en el juego de alto nivel; los resultados, tras el análisis de 3895 acciones, mostraron que el $25,3 \%$ se corresponden a pick and roll, y de las cuales sólo el 36,3\% de estas acciones se mostraron eficaces en el marcador, mientras que el $85,7 \%$ se mostraron eficaces tácticamente.

Esta técnica tiene su origen en el trabajo de Sackett (1980) y posterior optimización con la técnica de Anguera (1997) que permite efectuar una reducción drástica de datos y una representación vectorial de las interrelaciones establecidas entre las diferentes categorías que constituyen el sistema taxonómico propuesto (Gorospe y Anguera, 2000; HernándezMendo y Anguera, 1999). Esta técnica se apoya en un análisis secuencial de retardos prospectivo (Sackett, 1980) y retrospectivo, con la técnica genuina (Anguera, 1997) de las sucesivas conductas ocurridas. Los valores obtenidos en el cálculo de la probabilidad condicionada permitirán la obtención del parámetro Zsum $(Z s u m=\Sigma z / \sqrt{ }$ n, siendo $n$ el número de retardos) (Cochran, 1954). La distribución de este parámetro Zsum tiene una $=0 \mathrm{y}$ una $\mathrm{Sx}=1$.

A partir de la obtención de estos valores se puede construir el mapa interrelacional de conductas, o mapa de coordenadas polares (Gorospe y Anguera, 2000). Para la construcción de los mapas conductuales es necesario determinar los módulos de los vectores (para que se consideren significativas deberán ser iguales o superiores a 1,96). El Módulo o longitud del radio se calcula mediante la Raíz cuadrada de la suma del cuadrado de la Zsum de la X (prospectiva) y del cuadrado de la Zsum de la Y (retrospectiva).

$$
\text { Módulo }=\sqrt{x^{2}+y^{2}}
$$

El ángulo del vector $(\varphi)$ (que dependerá del cuadrante donde se sitúe) marcando la naturaleza de la relación (Castellano y Hernández-Mendo, 2003). Este ángulo $(\varphi)$ se calcula como $\varphi=$ Arco seno de Y/Radio. 


\section{Morillo-Baro et al.}

Teniendo en cuenta todos estos antecedentes sobre el baloncesto, el objetivo de esta investigación ha sido detectar, mediante un análisis de coordenadas polares, las relaciones que se establecen entre las conductas de finalización de los jugadores interiores de la Selección Española de Baloncesto y su repercusión en el rendimiento del equipo.

\section{MATERIAL Y MÉTODOS}

\section{Diseño}

El diseño utilizado fue nomotético, seguimiento y multidimensional, que se ubica en el IV cuadrante de los diseños observacionales (Anguera, BlancoVillaseñor, Hernández-Mendo y Losada, 2011). Nomotético, por el análisis de la pluralidad de los equipos: seguimiento, debido a la continuidad temporal en los diferentes partidos de clasificatoria; y multidimensional, con varias dimensiones que se corresponden con los distintos criterios del instrumento observacional. La unidad de observación fue la secuencia de ataque posicional durante el último cuarto de cada partido. La acción de registro estará definida desde que se atraviesa el centro del campo hasta que se realice un tiro a canasta, se cambie de posesión o se interrumpa el juego por una infracción reglamentaria.

\section{Participantes}

Se ha observado el último cuarto de cada uno de los 9 partidos que disputó la Selección Española de Baloncesto Masculina durante el Campeonato de Europa Senior que se celebró del 5 al 20 de septiembre de 2015. Se han registrado un total de 316 acciones ofensivas con sus correspondientes finalizaciones, pérdidas y/o interrupciones. A continuación, en la tabla 1 se muestra la relación de los partidos observados:

Tabla 1. Partidos analizados.

\begin{tabular}{lll}
\hline \multicolumn{1}{c}{ PARTIDO } & \multicolumn{2}{c}{ RESULTADO } \\
\hline $1^{\circ}$ FASE GRUPO & SERBIA 70 & - ESPAÑA 80 \\
\hline $2^{\circ}$ FASE GRUPO & ESPAÑA 104 - TURQUÍA 77 \\
\hline $3^{\circ}$ FASE GRUPO & ITALIA 98 - ESPAÑA 105 \\
\hline $4^{\circ}$ FASE GRUPO & ESPAÑA 99 & - ISLANDIA 71 \\
\hline $5^{\circ}$ FASE GRUPO & ESPAÑA 77 - ALEMANIA 76 \\
\hline OCTAVOS DE FINAL & ESPAÑA 80 - POLONIA 66 \\
\hline CUARTOS DE FINAL & ESPAÑA 73 & - GRECIA 71 \\
\hline SEMIFINAL & ESPAÑA 80 & - FRANCIA 75 \\
\hline FINAL & ESPAÑA 80 & - LITUANIA 63 \\
\hline
\end{tabular}

\section{Instrumentos}

Para realizar el registro y codificación de los datos, el análisis secuencial, el análisis de coordenadas polares y su representación vectorial se utilizó el software informático HOISAN (Hernández-Mendo, LópezLópez, Castellano, Morales-Sánchez y Pastrana, 2012). La herramienta de observación usada en esta investigación ha sido creada y diseñada ad hoc y ha superado las pruebas de calidad del dato exigibles en MO (Morillo-Baro y Hernández-Mendo, 2015). Está construida mediante un sistema mixto de formato de campo y sistemas de categorías, exhaustivas y mutuamente excluyentes (E/ME). Está compuesta por 5 criterios y 29 categorías que se corresponden con el desarrollo técnico-táctico de una acción ofensiva en baloncesto. La tabla 2 muestra la composición de la herramienta de observación.

Tabla 2. Listado de categorías correspondientes a cada criterio y sistema de codificación.

\begin{tabular}{|c|c|}
\hline Criterios & Categorías \\
\hline \multirow{6}{*}{$\begin{array}{l}\text { Jugador } \\
\text { Asistente }\end{array}$} & OASIST: Ningún jugador asiste \\
\hline & 1ASIST: asiste el base \\
\hline & 2ASIST: asiste el alero izquierdo \\
\hline & 3ASIST: asiste el alero derecho \\
\hline & 4ASIST: asiste el ala-pívot \\
\hline & 5ASIST: asiste el pívot \\
\hline \multirow{6}{*}{$\begin{array}{l}\text { Jugador } \\
\text { Finaliza }\end{array}$} & OFINAL: Ningún jugador lanza \\
\hline & 1FINAL: lanza el base \\
\hline & 2FINAL: lanza el alero izquierdo \\
\hline & 3FINAL: lanza el alero derecho \\
\hline & 4FINAL: lanza el ala pívot \\
\hline & 5FINAL: lanza el pívot \\
\hline \multirow{5}{*}{$\begin{array}{c}\text { Zona de } \\
\text { Finalización }\end{array}$} & ZF1: Finalización en la zona 1 \\
\hline & ZF2: Finalización en la zona 2 \\
\hline & ZF3: Finalización en la zona 3 \\
\hline & ZF4: Finalización en la zona 4 \\
\hline & ZF5: Finalización en la zona 5 \\
\hline \multirow{8}{*}{$\begin{array}{c}\text { Tipo de } \\
\text { Finalización }\end{array}$} & T.LIBRE: Finalización en tiro libre \\
\hline & $\begin{array}{l}\text { BANDEJA: Finalización en tiro sobre } \\
\text { tablero }\end{array}$ \\
\hline & T.SUSPENSIÓN: Finalización en \\
\hline & $\begin{array}{l}\text { lanzamiento de tiro en suspensión de } 2 \\
\text { puntos }\end{array}$ \\
\hline & MATE: Finalización en mate \\
\hline & TRIPLE: Finalización en lanzamiento \\
\hline & de tiro de 3 puntos \\
\hline & $\begin{array}{l}\text { FALTA DEFENSA: Falta del equipo } \\
\text { defensor }\end{array}$ \\
\hline
\end{tabular}




\section{El juego interior en el rendimiento del baloncesto}

\begin{tabular}{ll}
\hline & PÉRDIDA: Pérdida de balón \\
& INFRACCIÓN: Falta de ataque \\
& INTERRUPCIÓN: Final de tiempo de \\
& posesión, TM o final de cuarto \\
\hline \multirow{3}{*}{ Resultado } & ACIERTO: canasta \\
& NOACIERTO: no canasta \\
& NULO: no se produce lanzamiento \\
\hline
\end{tabular}

Para la concreción del criterio Zona de Finalización se ha dividido el campo de juego en 5 zonas distintas que van determinadas por la dirección del sentido del ataque, división que se muestra en la figura 1 .

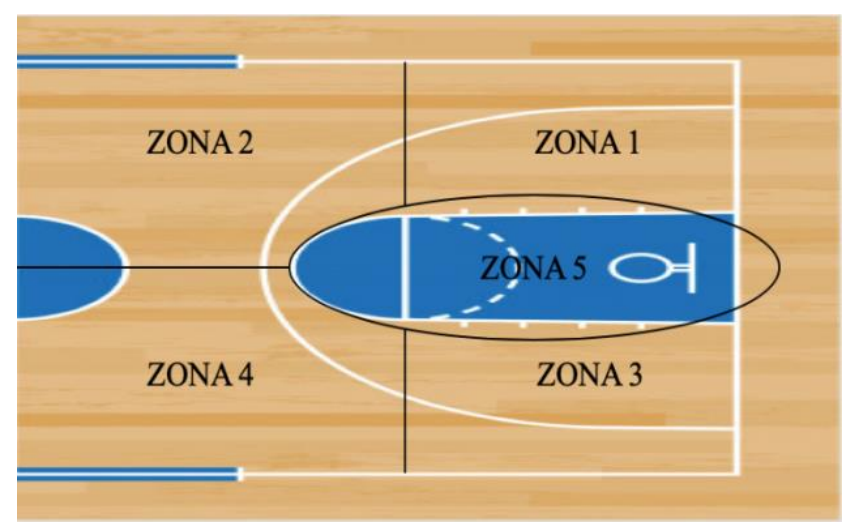

Figura 1. División de espacios de juego.

\section{Procedimiento}

El análisis de la Calidad del Dato se ha realizado desde dos diferentes orientaciones, la cualitativa y la cuantitativa (Blanco-Villaseñor, Losada y Anguera, 2003). Desde un punto de vista cualitativo se ha utilizado la concordancia consensuada (Anguera, 1990) con la finalidad de establecer un acuerdo previo entre los observadores en la creación del sistema de codificación, la elaboración del protocolo de observación y el entrenamiento exhaustivo de los observadores (Morillo y Hernández Mendo, 2015); y desde un punto de vista cuantitativo se han obtenido los índices de correlación de Pearson, Spearman y Tau-b de Kendall. En todos los casos los coeficientes de correlación superan el 0.90 , lo que supone unos resultados óptimos. Los resultados se muestran en la tabla 3.
Tabla 3. Resultados de los coeficientes de correlación Pearson, Spearman y Tau b de Kendall.

\begin{tabular}{ccc}
\hline \multicolumn{3}{c}{ COEFICIENTES DE CORRELACIÓN } \\
\hline COEFICIENTE & $\begin{array}{c}\text { CONCORDANCIA } \\
\text { INTRA }\end{array}$ & $\begin{array}{c}\text { CONCORDANCIA } \\
\text { INTER }\end{array}$ \\
\hline Pearson & 1.00 & 0.98 \\
Spearman & 1.00 & 0.99 \\
Tau-b de & 1.00 & 0.99 \\
Kendall & & \\
\hline
\end{tabular}

También desde la perspectiva cuantitativa, se ha calculado el índice de concordancia de Kappa de Cohen para cada uno de los criterios y para la sesión completa, estos resultados se muestran en la tabla 4.

Tabla 4. Resultados de los índices de kappa de Cohen en relación a los criterios y sesión completa.

\begin{tabular}{ccc}
\hline \multicolumn{3}{c}{ ÍNDICES DE KAPPA DE COHEN } \\
\hline Criterios & $\begin{array}{c}\text { Concordancia } \\
\text { INTRA }\end{array}$ & $\begin{array}{c}\text { Concordancia } \\
\text { INTER }\end{array}$ \\
\hline $\begin{array}{c}\text { JUGADOR } \\
\text { ASISTENTE }\end{array}$ & 1.00 & 1.00 \\
\hline $\begin{array}{c}\text { JUGADOR } \\
\text { FINALIZA }\end{array}$ & 1.00 & 0.93 \\
\hline $\begin{array}{c}\text { ZONA DE } \\
\text { FINALIZACIÓN }\end{array}$ & 1.00 & 1.00 \\
\hline $\begin{array}{c}\text { TIPO DE } \\
\text { FINALIZACIÓN }\end{array}$ & 1.00 & 1.00 \\
\hline RESULTADO & 1.00 & 1.00 \\
\hline $\begin{array}{c}\text { SESIÓN } \\
\text { COMPLETA }\end{array}$ & 1.00 & 0.98 \\
\hline
\end{tabular}

Como se puede observar, el índice de Kappa de Cohen para esta investigación supera el 0.90 tanto para la fiabilidad intraobservador (1.00) como para la interobservador (0.98). Estos resultados muestran una alta fiabilidad atendiendo a los valores propuestos por Gelfand y Hartmamm (1975) y Landis y Koch (1977).

Para la codificación y análisis de Coordenadas Polares, se ha usado el programa informático HOISAN (Hernández-Mendo et al., 2012). En primer lugar, se realizó un análisis secuencial para cada categoría de todas las observaciones realizadas con la conducta criterio seleccionada, obteniendo los resultados $Z$ con un rango de retardos de $-5 \mathrm{y}+5$. Una vez obtenidos estos valores se realizaron los cálculos para determinar los parámetros Zsum (prospectivo y 


\section{Morillo-Baro et al.}

retrospectivo), la asignación de cuadrante, el módulo, el ángulo, y el ángulo transformado (AT) para el resto de categorías (Castellano y Hernández-Mendo, 2003). La caracterización de cada cuadrante es la siguiente (Castellano y Hernández-Mendo, 2003):

Cuadrante I $[+,+]$ : La conducta criterio se excita con respecto a la conducta de apareo en perspectiva retrospectiva y prospectiva.

Cuadrante II $[-,+]$ : La conducta criterio tiene una relación con respecto a la de apareo de excitación en perspectiva retrospectiva y de inhibición en perspectiva prospectiva.

Cuadrante III [-,-]: La conducta criterio tiene una relación con respecto a la de apareo de inhibición en perspectiva retrospectiva y prospectiva.

Cuadrante IV [+,-]: La conducta criterio tiene una relación con la conducta de apareo de excitación en perspectiva prospectiva y de inhibición en perspectiva retrospectiva.

Las conductas focales utilizadas para realizar el análisis de coordenadas polares han sido las siguientes:
- 4FINAL y 5FINAL: finaliza la acción de ataque el ala-pívot (4FINAL) y el pívot (5FINAL).

- ACIERTO y NOACIERTO: la acción de ataque finaliza en canasta (ACIERTO) y la acción finaliza en tiro fallado (NOACIERTO).

La decisión sobre la elección de las conductas focales viene determinada por el objetivo del estudio, la identificación de la repercusión del juego interior en el resultado del partido.

\section{RESULTADOS}

Los resultados de los análisis de coordenadas polares se muestran por las parejas de conductas focales seleccionadas; el jugador que finaliza: 4FINAL (alapívot) y 5FINAL (pívot) y el resultado de las acciones: ACIERTO y NOACIERTO.

A continuación, en la tabla 5 se muestran los resultados para la primera pareja de conductas focales seleccionadas.

Tabla 5. Relaciones significativas encontradas con las conductas focales 4FINAL y 5FINAL.

\begin{tabular}{|c|c|c|c|c|c|c|}
\hline \multirow{2}{*}{$\mathbf{C}$} & \multicolumn{3}{|c|}{ 4FINAL ALA-PIVOT } & \multicolumn{3}{|c|}{ 5FINAL PIVOT } \\
\hline & C. APAREO & MÓDULO & ÁNGULO T. & C. APAREO & MÓDULO & ÁNGULO T. \\
\hline \multirow{6}{*}{ I } & T.LIBRE & 5.12 & 23.75 & ZF5 & 5.35 & 42.89 \\
\hline & OASIST & 3.27 & 2.88 & 4ASIST & 4.65 & 72.64 \\
\hline & & & & T.LIBRE & 4.47 & 46.48 \\
\hline & & & & T.SUSPENSIÓN & 4.28 & 50.91 \\
\hline & & & & ACIERTO & 4.00 & 40.10 \\
\hline & & & & OASIST & 2.72 & 54.37 \\
\hline \multirow{4}{*}{ II } & FALTA DEF & 2.49 & 173.42 & & & \\
\hline & NULO & 2.33 & 165.09 & & & \\
\hline & 1ASIST & 2.06 & 173.53 & & & \\
\hline & TRIPLE & 1.96 & 127.97 & & & \\
\hline \multirow{9}{*}{ III } & T.SUSPENSIÓN & 2.67 & 237.25 & $\mathrm{ZF} 1$ & 4.40 & 213.44 \\
\hline & BANDEJA & 2.45 & 234.4 & PÉRDIDA & 3.84 & 203.19 \\
\hline & ZF1 & 2.05 & 187.61 & INTERRUPCIÓN & 3.71 & 254.71 \\
\hline & & & & NULO & 3.47 & 214.70 \\
\hline & & & & 3ASIST & 3.39 & 248.16 \\
\hline & & & & TRIPLE & 3.24 & 230.65 \\
\hline & & & & 1ASIST & 2.71 & 220.98 \\
\hline & & & & $\mathrm{ZF} 2$ & 2.52 & 234.65 \\
\hline & & & & $\mathrm{ZF} 4$ & 2.46 & 194.60. \\
\hline \multirow{2}{*}{ IV } & ACIERTO & 3.28 & 358.33 & 2ASIST & 2.16 & 277.25 \\
\hline & ZF5 & 2.24 & 357.21 & BANDEJA & 2.07 & 272.56 \\
\hline
\end{tabular}




\section{El juego interior en el rendimiento del baloncesto}

En el cuadrante I, donde las relaciones que se establecen son de mutua excitación, destaca la coincidencia de las conductas de apareo tiro libre (T.LIBRE) y nadie asiste (0ASIST) para las dos conductas focales seleccionadas. Igualmente destacan, con la conducta focal que determina las finalizaciones del pívot, las conductas de apareo acierto y la zona de finalización 5, que se corresponde con la zona dentro de la zona.

En el segundo cuadrante no se presentan relaciones significativas con la conducta focal finalización del pívot, sin embargo, sí aparecen con el ala-pívot, destacando estas relaciones de inhibición en la perspectiva prospectiva y excitación en la retrospectiva las conductas 1ASIST (asistencias del base) y las faltas de los defensas.

En el tercer cuadrante, donde las relaciones que se establecen son de mutua inhibición, destaca el mayor número de conductas significativas que se asocian a la conducta focal finaliza el pívot. Las conductas de apareo pertenecen, principalmente, a los criterios zona y tipo de finalización, destacando la conducta pérdida de la posesión. Para la conducta focal ala-pívot destaca el tipo de finalización mediante bandeja y tiro en suspensión.

Por último, en el cuadrante IV, ambas conductas focales se relacionan de forma significativa con dos conductas de apareo. Estas relaciones son de excitación en la perspectiva prospectiva y de inhibición en la retrospectiva, destacando la conducta focal acierto para la conducta focal ala-pívot.

La figura 2 muestra la representación gráfica resultante del análisis de coordenadas polares para estas conductas focales.

Figura 2. Representación gráfica del análisis de coordenadas polares

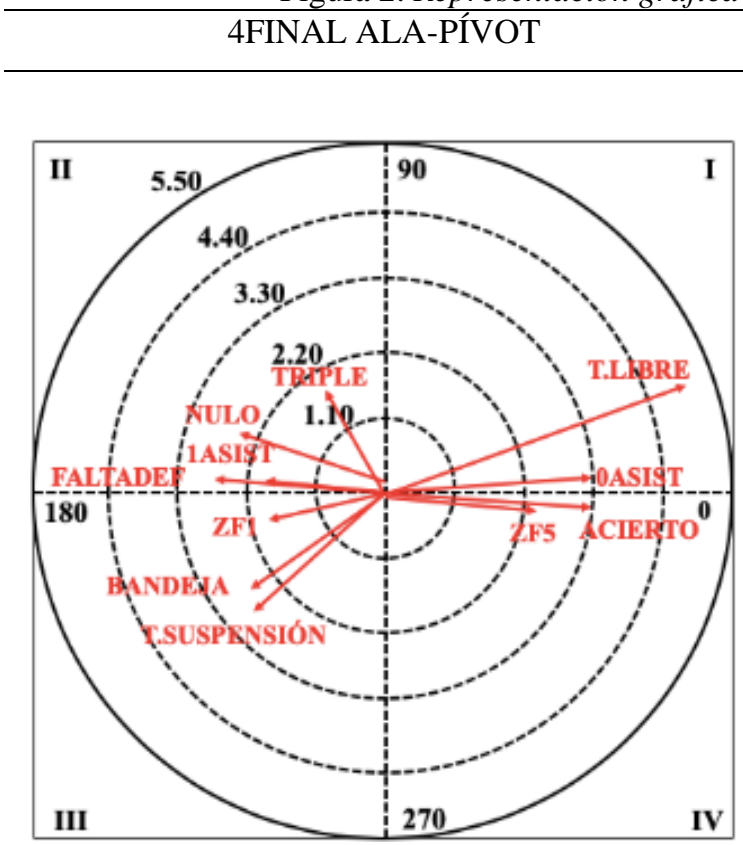
5FINAL PÍVOT

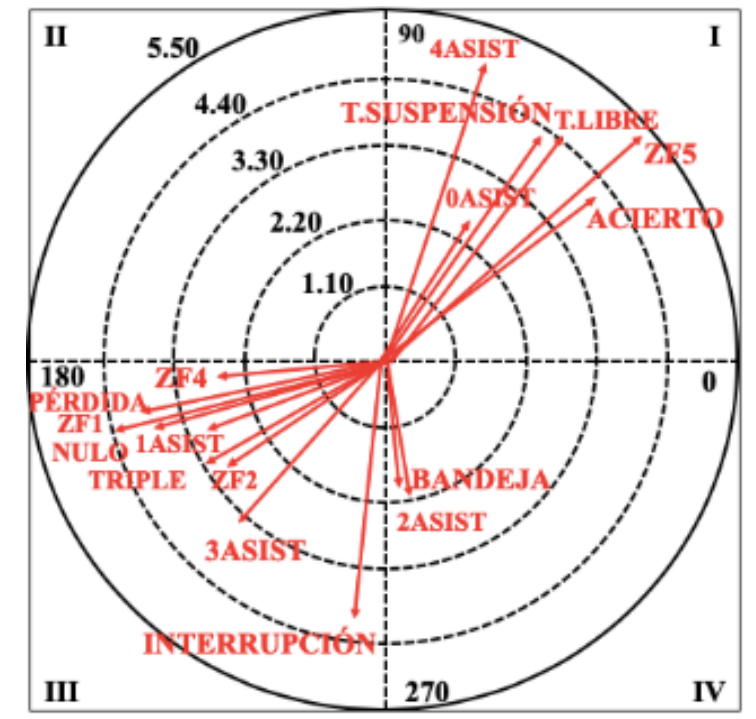

En la tabla 6 se muestran los resultados del análisis de coordenadas polares realizados con las conductas focales acierto y no acierto en la finalización de las acciones de los jugadores. 


\section{Morillo-Baro et al.}

Tabla 6. Relaciones significativas encontradas con las conductas focales ACIERTO y NOACIERTO.

\begin{tabular}{|c|c|c|c|c|c|c|}
\hline \multirow[b]{2}{*}{$\mathbf{C}$} & \multicolumn{3}{|c|}{ ACIERTO } & \multicolumn{3}{|c|}{ NOACIERTO } \\
\hline & C. APAREO & MÓDULO & ÁNGULO T. & C. APAREO & $\begin{array}{l}\text { MÓDU } \\
\text { LO }\end{array}$ & ÁNGULO T. \\
\hline \multirow{6}{*}{ I } & 5FINAL & 4.00 & 49.90 & 1ASIST & 3.69 & 10.52 \\
\hline & OASIST & 3.91 & 38.95 & BANDEJA & 2.14 & 75.19 \\
\hline & ZF5 & 3.38 & 40.85 & $\mathrm{ZF} 2$ & 2.04 & 4.85 \\
\hline & $\mathrm{ZF} 4$ & 2.55 & 55.24 & TRIPLE & 2.00 & 33.36 \\
\hline & 4ASIST & 2.50 & 15.12 & & & \\
\hline & T.SUSPENSIÓN & 2.10 & 32.28 & & & \\
\hline \multirow{3}{*}{ II } & ZF3 & 3.69 & 116.69 & MATE & 3.12 & 106.55 \\
\hline & FALTA DEF & 3.38 & 173.60 & ZF1 & 2.56 & 102.07 \\
\hline & 4 FINAL & 3.28 & 91.67 & & & \\
\hline \multirow{9}{*}{ III } & ZF1 & 5.55 & 237.37 & T.LIBRE & 3.85 & 201.70 \\
\hline & 0FINAL & 4.58 & 257.18 & INTERRUPCIÓN & 3.28 & 225.67 \\
\hline & PÉRDIDA & 4.00 & 220.60 & ZF5 & 3.06 & 189.12 \\
\hline & $\mathrm{ZF} 2$ & 3.94 & 251.34 & & & \\
\hline & 3FINAL & 3.75 & 259.67 & & & \\
\hline & $5 \mathrm{ASIST}$ & 3.41 & 191.87 & & & \\
\hline & 1ASIST & 3.07 & 220.35 & & & \\
\hline & BANDEJA & 2.68 & 259.41 & & & \\
\hline & 1FINAL & 1.99 & 182.52 & & & \\
\hline \multirow{4}{*}{ IV } & MATE & 3.00 & 307.37 & ZF3 & 3.04 & 351.94 \\
\hline & INTERRUPCIÓN & 2.14 & 282.49 & FALTA DEF & 2.02 & 326.69 \\
\hline & & & & T.SUSPENSIÓN & 2.00 & 323.75 \\
\hline & & & & 3ASIST & 1.96 & 274.67 \\
\hline
\end{tabular}

Para ambas conductas focales, los resultados muestran conductas de apareo diferentes en todos los cuadrantes, destacando en el primero los diferentes jugadores que realizan la asistencia, la zona de finalización y el tipo. La conducta focal acierto se relaciona significativamente con una mutua excitabilidad tanto en la perspectiva prospectiva como retrospectiva con las zonas del terreno de juego 4 y 5 , con la finalización del pívot, las asistencias del alapívot o sin ella y el tiro en suspensión; por su parte, las conductas de no acierto se relacionan en este primer cuadrante con las asistencias del base, el tipo de finalización en bandeja o triple y la zona 2 .

En el segundo cuadrante se presentan menor número de relaciones significativas con ambas conductas focales. Las relaciones en este cuadrante son de inhibición en la perspectiva prospectiva y excitación en la retrospectiva, concretándose en este estudio en las conductas zona de finalización 3, falta defensiva y finaliza el ala-pívot para la conducta focal acierto; mientras que la finalización mediante el gesto técnico del mate y la zona 1 para la conducta focal no acierto.

El cuadrante III muestra las relaciones de mutua inhibición entre la conducta focal seleccionada y las conductas de apareo. Para la conducta focal acierto se observan relaciones significativas: asistencias del base (1ASIST) y asistencias del pívot (5ASIST), el base finaliza (1FINAL), alero finaliza (3FINAL) y nadie finaliza (OFINAL), las zonas de lanzamiento 1 (ZF1) y 2 (ZF2) y los tipos de finalización BANDEJA y PÉRDIDA. Para la otra conducta focal, no acierto, las relaciones significativas se han mostrado con: interrupción, tiro libre y la zona 5 (ZF5).

Por último, en el cuadrante IV donde las relaciones son de excitación en la perspectiva prospectiva y de inhibición en la retrospectiva, destacan para la conducta focal acierto la finalización en mate y la interrupción; mientras que para la conducta focal no acierto el alero realiza la asistencia (3ASIST), la zona 


\section{El juego interior en el rendimiento del baloncesto}

de lanzamiento 3 (ZF3), el tiro en suspensión y la falta defensiva.
La figura 3 muestra la representación gráfica resultante del análisis de coordenadas polares para estas conductas focales.

Figura 3. Representación gráfica del análisis de coordenadas polares

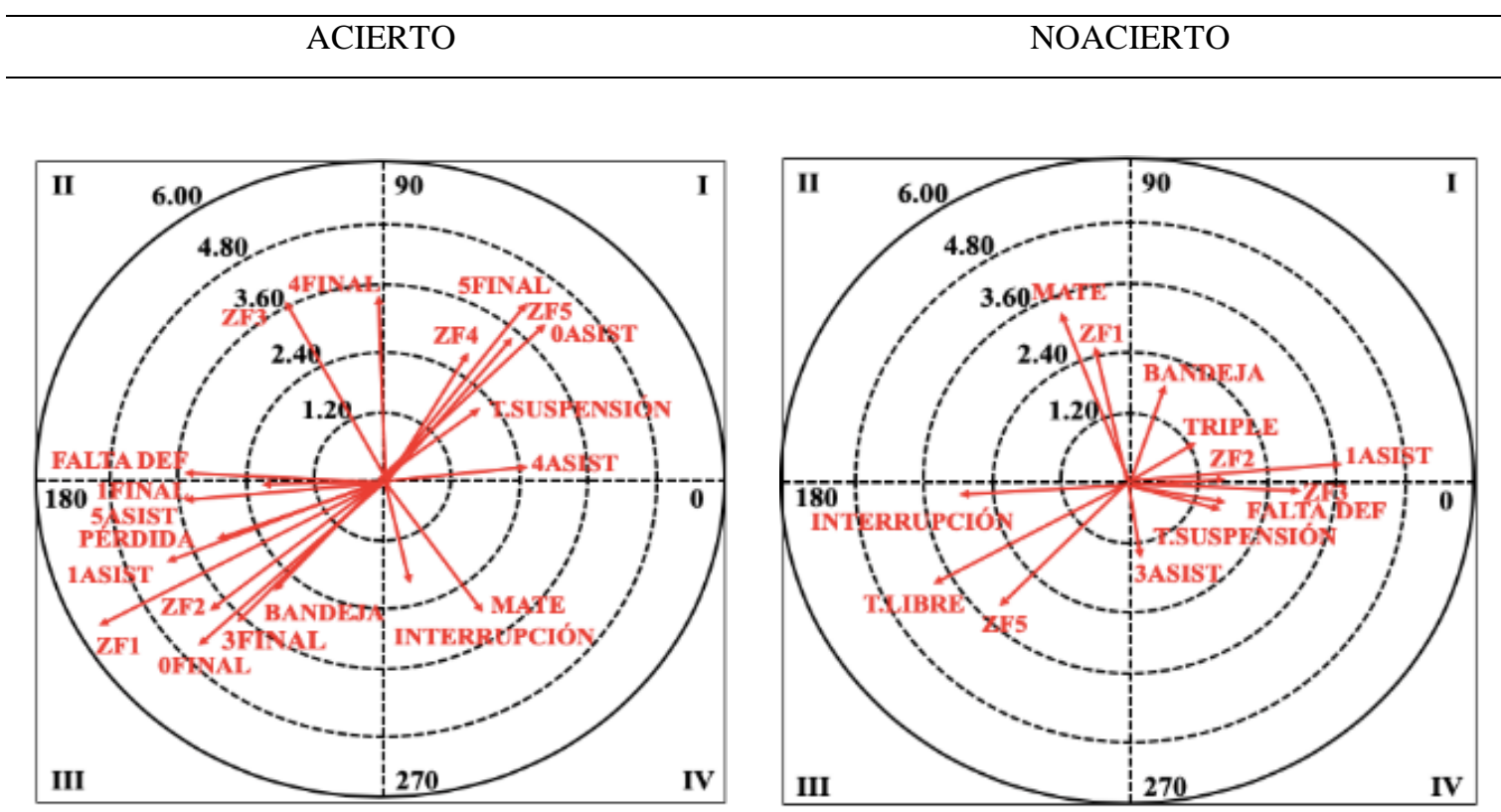

\section{DISCUSIÓN}

El objetivo de este estudio fue identificar las relaciones estadísticamente significativas entre las conductas de finalización de los jugadores pivot y alapivot, seleccionadas como focales, y el resto de conductas de apareo de la herramienta de observación usada, lo cual ha permitido interpretar la influencia del juego interior en el ataque estático de la Selección Española de Baloncesto durante el Campeonato de Europa de 2015. Para ello, se realizaron varios análisis de coordenadas polares que han puesto de manifiesto las relaciones entre las conductas estudiadas, mostrándose como una técnica muy útil para el análisis táctico de las situaciones de juego en competición.

En primer lugar, la herramienta de observación creada ha superado las pruebas del análisis de Calidad del Dato realizadas, requisito indispensable para poder realizar un registro fiable de las conductas que aparecen durante las acciones de juego (Vázquez-Diz, Morillo-Baro, Reigal, Morales-Sánchez y Hernández-
Mendo, 2019c; Quiñones et al., 2019). Se decidió registrar las acciones del último cuarto del partido, ya que es donde hay una mayor tensión y se ponen en juego a los mejores jugadores de cada equipo, con el objetivo final de conseguir la victoria (Sampaio, Lorenzo y Ribero, 2006). Además, en ese cuarto normalmente se ubica el momento crítico del partido, como son los últimos 5 minutos y la diferencia entre ambos equipos está por debajo de 6 puntos (Navarro, Gómez-Ruano, Lorenzo-Calvo, Lorenzo-Calvo y Jiménez-Saiz, 2012).

La realización de este estudio ha permitido obtener información sobre las acciones ofensivas usadas por parte de la Selección Española de Baloncesto cuando finalizan los jugadores interiores, donde están sus jugadores más determinantes a la hora de incidir sobre el resultado final. En este sentido, los resultados muestran diferencias en las relaciones estadísticamente significativas entre las conductas focales, según finalice el ala-pívot o el pívot, y el resto de conductas de apareo. 


\section{Morillo-Baro et al.}

Cuando finaliza el ataque estático el ala-pívot, lo suele hacer consiguiendo canasta y sin necesidad de que lo asista nadie, ya que las conductas que se excitan en el Cuadrante I son las conductas de apareo del tiro libre y nadie le asiste. Relaciones que se refuerzan con las conductas de apareo que aparecen en el Cuadrante IV, ya que se puede interpretar que los ataques que finaliza este jugador suelen acabar en canasta y en la zona de finalización más cercana al aro, donde suelen pasar la mayor parte del tiempo los jugadores interiores, si bien es cierto que la tendencia en los últimos años está cambiando, y cada vez son más los jugadores interiores capaces de realizar tiros en posiciones exteriores (Rolland, Vuillemot, Bos y Rivière, 2020).

Por otro lado, cuando el jugador que finaliza es el pívot, nuevamente lo hace en zonas cercanas al aro, igualmente sin necesidad de lo que lo asista nadie o, si lo hace algún compañero, es el ala-pívot; y es capaz de conseguir canasta mediante tiro en suspensión o con un tiro. Todas estas conductas aparecen en el Cuadrante I donde la relación es de mutua excitación entre la conducta focal y la de apareo. Las conductas de apareo que aparecen en el Cuadrante III respaldan esta interpretación de acierto en la finalización del pívot, ya que se inhiben las conductas de pérdida del balón y finalización nulo del ataque; además, también se inhiben conductas de zonas de finalización lejanas al aro y los tiros de 3 puntos. Por último, la aparición en el Cuadrante IV de la conducta tipo de finalización mediante bandeja, tiro que se realiza en aproximación a la canasta, incide nuevamente sobre la cercanía de este jugador al aro.

La presencia de jugadores de tan reconocido prestigio internacional, debido entre otras a las cualidades técnico-tácticas que poseen, permite interpretar la búsqueda incesante del juego interior por parte de la selección nacional española, beneficiándose así de la capacidad de liderazgo y anotación de Pau Gasol, uno de los jugadores más determinantes en el panorama europeo en la última década y que resultó máximo anotador y jugador más valioso del campeonato.

Los resultados del análisis de coordenadas polares que se han obtenido para las conductas focales acierto y no acierto, refuerzan la interpretación de los resultados expuestos hasta ahora. Por ejemplo, para la conducta focal acierto, en el Cuadrante I se excitan las conductas de apareo de finalización del pívot, sin la asistencia de algún compañero, en zonas cercanas al aro y mediante tiro en suspensión. Además, en el Cuadrante II, donde la conducta focal excita la aparición de la conducta de apareo en la perspectiva retrospectiva, se excitan la finalización del ala-pívot y la falta defensiva. En cuanto a la finalización en tiro errado, en el Cuadrante I se excita la conducta de tiro de 3 puntos, por lo que se puede interpretar que la finalización por zonas interiores produce un mayor acierto en los ataques estáticos de la Selección.

La interpretación de todos estos resultados apunta hacia una finalización exitosa de los jugadores interiores, consiguiendo canastas o forzando faltas a los defensores que actúan sobre ellos. Aunque, como se ha comentado anteriormente, la tendencia es que cada vez más los jugadores interiores finalicen lejos del aro y realicen un mayor número de tiros de 3 puntos, este hecho no ha influido, al menos durante este campeonato, en el juego de los jugadores interiores de la Selección y que tienen su zona de influencia más óptima cerca de la canasta. Se han mostrado jugadores capaces de buscarse sus propios tiros, sin necesidad de la asistencia de sus compañeros ni procedimientos ofensivos específicos que les creen alguna ventaja, ni siquiera el uso del pick and roll, procedimiento táctico más utilizado en el ataque estático en baloncesto (Marmarinos, Apostolidis, Kostopoulos y Apostolidis, 2016).

En conclusión, los resultados han puesto de manifiesto la importancia que tuvieron los jugadores interiores en el ataque estático de la Selección Española de Baloncesto y que los llevó a conseguir la medalla de oro en el Campeonato de Europa de 2015. Así, el uso de la observación y la técnica de Coordenadas Polares han sido útiles para discriminar entre acciones y comportamientos específicos, lo cual proporciona datos sobre las conductas realizadas por los deportistas durante la competición. Este tipo de análisis ha permitido detectar las relaciones estadísticamente significativas de las conductas focales elegidas con las diferentes conductas de apareo que se corresponden con las distintas facetas del juego.

Las limitaciones que presenta este estudio se podrían centrar la imposibilidad de extrapolar o generalizar los resultados a otros campeonatos, ya que tanto la composición de la plantilla de jugadores como su propio estado de forma varía de un evento a otro. Además, no se podría definir por completo el modelo de juego de la Selección al basarse el análisis en la 


\section{El juego interior en el rendimiento del baloncesto}

influencia únicamente del juego interior. Por lo que sería interesante seguir esta línea de investigación y analizar tácticamente otros puestos específicos, incluso con herramientas de observación que profundicen más desde el punto de vista técnicotáctico del juego. Finalmente, analizar únicamente el último cuarto de cada partido podría ser también una limitación en aquellos partidos en los que la diferencia de puntos sea muy notable al comenzar este cuarto.

\section{APLICACIONES PRÁCTICAS}

Este estudio permite a los entrenadores y preparadores de equipos de élite conocer las posibles conductas que ocurren durante el juego interior y la importancia de las acciones de estos jugadores en el rendimiento. Además, permite obtener rastros de conductas de los sistemas de juego con el objetivo de mejorar la eficacia de estas acciones en competición. Todo ello da la oportunidad de diseñar y aplicar de forma previa, durante el entrenamiento, las medidas para contrarrestar la táctica del contrario.

\section{REFERENCIAS (APA $7^{\mathrm{a}}$ EDICION)}

1. Anguera, M. T. (1990). Metodología observacional. En: Arnau, J., Anguera M. T. y Gómez J. B. (Eds), Metodología de la investigación en las Ciencias del Comportamiento, 125-238. Murcia: Universidad de https://doi.org/10.18239/ensayos.v35i1.2332

2. Anguera, M. T. (1997). From prospective patterns in behavior to joint analysis with a retrospective perspective. Colloque sur invitation «Méthodologie d'analyse des interactions sociales». París: Université de la Sorbonne.

3. Anguera, M. T., Blanco-Villaseñor, A., Hernández-Mendo, A. y Losada, J. L. (2011). Diseños observacionales: ajuste y aplicación en psicología del deporte. Cuadernos de Psicología del Deporte, vol. 11, 2, 63-76. https://doi.org/10.4321/s1578-

84232015000100002

4. Anguera, M. T. y Hernández-Mendo, A. (2013). La metodología observacional en el ámbito del deporte. E-balonmano.com: Revista de Ciencias del Deporte, vol. 9, 3, 135-160. https://doi.org/10.4321/s157884232015000100002

5. Blanco-Villaseñor, A., Losada, J. L. y Anguera, M. T. (2003). Data analysis techniques in observational designs applied to the environmentbehaviour relation. Medio ambiente $y$ comportamiento humano, vol. 4, 2, 111-126.

6. Castañer, M., Barreira, D., Camerino, O., Anguera, M. T., Canton, A. e Hileno, R. (2016). Goal scoring in soccer: a polar coordinate analysis of motor skills used by Lionel Messi. Frontiers in Psychology, $7: 806$ https://doi.org/10.3389/fpsyg.2016.00806

7. Castellano, J. y Hernández-Mendo, A. (2003). El análisis de coordenadas polares para la estimación de relaciones en la interacción motriz en fútbol. Psicothema, vol. 15, 4, 569-574. https://doi.org/10.4321/s157884232015000100017

8. Castro, C. A. (2018). Propuesta de análisis táctico deportivo con herramientas tecnológicas aplicadas a jugadores de fútbol profesional del Club Independiente Santa Fe. https://doi.org/10.31910/rdafd.v5.n1.2019.1123

9. Cochran, W. G. (1954). Some methods for strengthening the common $\chi^{2}$ tests. Biometrics, 10, 417-451. https://doi.org/10.2307/3001616

10. Conejero, M., Claver, F., Fernández-Echevarría, C., Gil-Arias, A. y Moreno, M. P. (2016). Diseño y validación de un instrumento de observación para valorar la toma de decisiones en la acción de recepción en voleibol. Cultura, Ciencia y Deporte, vol. $\quad 12, \quad 34, \quad 67-75$. https://doi.org/10.12800/ccd.v12i34.833

11. García-Santos, D. e Ibáñez, S. J. (2016). Diseño y validación de un instrumento de observación para la valoración de un árbitro de baloncesto (IOVAB). SPORT TK-Revista Euroamericana de Ciencias del Deporte, vol. 5, 2, 15-26. https://doi.org/10.6018/264601 


\section{Morillo-Baro et al.}

12. Gelfand, D. M y Hartmann, D. P. (1975). Child behavior analysis and therapy. Michigan: Pergamon Press.

13. Gorospe, G. y Anguera, M. T. (2000). Modificación de la técnica clásica de coordenadas polares mediante un desarrollo distinto de la retrospectividad: Aplicación al tenis. Psicothema, vol. 12, 2, 279-282.

14. Hernández-Mendo, A. y Anguera, M. T. (1999). Aportaciones de análisis de coordenadas polares a los deportes de equipo. En F. Guillén (Ed.), La Psicología del Deporte en España al final del milenio, 169-175. Las Palmas: Universidad de Las Palmas de Gran Canaria. https://doi.org/10.25145/b.emprendimientouni.20 20

15. Hernández-Mendo, A., López-López, J., Castellano, J., Morales-Sánchez, V. y Pastrana, J. L. (2012). Hoisan 1.2: Programa informático para uso en metodología observacional. Cuadernos de Psicología del Deporte, vol. 12, 1, 55-78. https://doi.org/10.4321/s1578-

84232012000100006

16. Landis, J. R. y Koch, G. G. (1977). The measurement of observer agreement for categorical data. Biometrics, 33, 159-174. https://doi.org/10.2307/2529310

17. López-López, J. A., Menescardi, C., Estevan, I., Falcó, C. y Hernández-Mendo, A. (2015). Análisis técnico-táctico en Taekwondo con coordenadas polares a través del software HOISAN. Cuadernos de Psicología del Deporte, vol. $15, \quad 1, \quad 131 \quad-142$. https://doi.org/10.4321/s157884232015000100013

18. Marmarinos, C., Apostolidis, N., Kostopoulos, N., y Apostolidis, A. (2016). Efficacy of the "pick and roll" offense in top level European basketball teams. Journal of human kinetics, vol. 51, 1, 121129. https://doi.org/10.1515/hukin-2015-0176

19. Morales, S., Lorenzo, A. y Concepción, R. R. (2008). Estudio de variables clave para el análisis del control del rendimiento técnico-táctico del voleibol de alto nivel. Lecturas: educación física y deportes, vol. 121, 7 . https://doi.org/10.46642/efd.v25i271.2670

20. Moreno, E. y Gómez, M. A. (2017). Validación herramienta observacional para el análisis de rachas de lanzamiento en baloncesto. Revista de Psicología del Deporte, vol. 26, 1, 87-93. https://doi.org/10.20868/upm.thesis.48327

21. Morillo-Baro, J. P. y Hernández Mendo, A. (2015). Análisis de la calidad del dato de un instrumento para la observación del ataque en balonmano playa. Revista Iberoamericana de Psicología del Ejercicio y el Deporte, vol. 10, 1, 15-22. https://doi.org/10.6018/cpd.423051

22. Morillo-Baro, J. P., Reigal, R. E. y HernándezMendo, A. (2015). Análisis del ataque posicional de balonmano playa masculino y femenino mediante coordenadas polares. Revista Internacional de Ciencias del Deporte, 11, 226 244. https://doi.org/10.5232/ricyde2015.04103

23. Muñoz-Arroyave, V., y Serna, J. (2015). Diseño, fiabilidad y validez del instrumento de observación SOCCB para el análisis de las finalizaciones en baloncesto. Cuadernos de Psicología del Deporte, vol. 15, 3, 169-174. https://doi.org/10.4321/s157884232015000300017

24. Muñoz-Arroyave, V., Serna, J., Daza, J. e Hileno, R. (2015). Influencia del bloqueo directo y el uno contra uno en el éxito del lanzamiento en baloncesto. Apuntes Educación Física y Deporte, 199, 80-86. https://doi.org/10.5672/apunts.20140983.es.(2015/1).119.05

25. Navarro, R. M., Gómez-Ruano, M. A., LorenzoCalvo, J., Lorenzo-Calvo, A. y Jiménez-Sáiz, S. (2012). La influencia del "Home Advantage" en el resultado de los momentos críticos en los partidos de baloncesto. Revista Española de Educación Física y Deportes, vo.1 396, 49-64. https://doi.org/10.5672/apunts.20140983.es.(2012/1).107.10

26. Nunes, H., Iglesias, X., Daza, G., Irrutia, A., Caparrós, T. y Anguera, M. T. (2015). Influencia 


\section{El juego interior en el rendimiento del baloncesto}

del pick and roll en el juego de ataque en baloncesto de alto nivel. Cuadernos de Psicología del Deporte, vol. 16, 1, 129-142. https://doi.org/10.4321/s1578-

84232015000100015

27. Ortín, F. J., Olmedilla, A. y Lozano, F. J. (2003). La utilización de registros para la mejora del comportamiento táctico en deportistas de equipo. Revista de Psicología del Deporte, vol. 12,1, 95105. https://doi.org/10.12800/ccd.v4i10.130

28. Quiñones, Y., Morillo-Baro, J. P., Reigal, R. E., Morales-Sánchez, V., Vázquez-Diz, J. A., y Hernández-Mendo, A. (2019). El ataque posicional en balonmano: validación de un sistema de observación. Cuadernos de Psicología del Deporte, 19(3), 114-124. https://doi.org/10.6018/cpd.384091

29. Quiñones, Y., Morillo-Baro, J. P., Reigal, R. E., Morales-Sánchez, V., Vázquez-Diz, J. A. y Hernández-Mendo, A. (2020). El juego combinativo ofensivo en el balonmano de élite: diferencias por género mediante análisis de coordenadas polares. Cuadernos de Psicología del Deporte, 20(1), 86-102. https://doi.org/10.6018/cpd.398741

30. Rolland, G., Vuillemot, R., Bos, W. y Rivière, N. (2020). Characterization of Space and TimeDependence of 3-Point Shots in Basketball. In MIT Sloan Sports Analytics Conference.

31. Romarís, I. U., Refoyo, I. y Coterón, J. (2013). La finalización de las posesiones en baloncesto: estudio de la acción de finalización. Cuaderno de Psicología del Deporte, vol. 12, 1, 45-50. https://doi.org/10.4321/s157884232012000300011

32. Sackett, G. P. (1980). Lag sequential analysis as a data reduction technique in social interaction research. En D.B. Sawin, R.C. Hawkins, L.O. Walker y J.H.

33. Sampaio, J., Lorenzo, A., y Ribero, C. (2006). Momentos críticos en los partidos de baloncesto: metodología para la identificación y análisis de los acontecimientos precedentes. Cultura, ciencia y deporte, vol. $\quad 2, \quad 5, \quad 83-88$. https://doi.org/10.12800/ccd.v2i5.177

34. Sarmento, H., Clemente, P. M., Araújo, D., Davids, K., McRobert, A. y Figueiredo, A. (2017). What performance analysts need to know about research trens in Association Football (20122016). Sports Medicine. https://doi.org/10.1007/s40279-017-0836-6

35. Suárez-Cadenas, E., Courel, J. y Cárdenas, D. (2017). La toma de decisiones en baloncesto. Una propuesta de árboles decisionales para la enseñanza del bloqueo directo. Acción psicológica, vol. $14, \quad 1, \quad 43-56$. https://doi.org/10.5944/ap.14.1.19259

36. Vázquez-Diz, J. A., Morillo-Baro, J. P., Reigal, R. E., Morales-Sánchez, V. y Hernández-Mendo, A. (2019a). Mixed Methods in Decision-Making Through Polar Coordinate Technique: Differences by Gender on Beach Handball Specialist. Frontiers in Psychology. 10:1627. https://doi.org/10.3389/fpsyg.2019.01627

37. Vázquez-Diz, J. A., Morillo-Baro, J. P., Reigal, R. E., Morales-Sánchez, V. y Hernández-Mendo, A. (2019b). Contextual Factors and Decision-Making in the Behavior of Finalization in the Positional Attack in Beach Handball: Differences by Gender Through Polar Coordinates Analysis. Frontiers in Psychology https://doi.org/10.3389/fpsyg.2019.01386

38. Vázquez-Diz, J. A., Morillo-Baro, J. P., Reigal, R. E., Morales-Sánchez, V. y Hernández-Mendo, A. (2019c). Diseño y validación de una herramienta de observación para porteros en balonmano playa. Cuadernos de Psicología del Deporte, 19(2), 135146. https://doi.org/10.6018/cpd.368901 